\title{
Growth of graphene and graphite nanocrystals from a molten phase
}

\author{
Shaahin Amini • Haamun Kalaantari • \\ Javier Garay • Alexander A. Balandin • \\ Reza Abbaschian
}

Received: 22 November 2010/ Accepted: 2 March 2011/Published online: 11 March 2011

(C) The Author(s) 2011. This article is published with open access at Springerlink.com

\begin{abstract}
We report on the development of a new technique for the growth of graphene and graphite nanocrystals from a metal-carbon melt. The process involves dissolving carbon inside molten nickel or copper at a specified temperature and then allowing the dissolved carbon to nucleate and grow on top of the melt at a lower temperature. The morphology of graphite forming inside nickel is shown to depend on the melt composition and cooling rate. The results indicate that the graphene layers contain a wrinkled structure due to thermal expansion coefficient mismatch between the grown layers and substrate. The graphene layers, however, preserve their continuity over these wrinkles. Although the formation of wrinkles was found not to depend on the type of metal substrate, the substrate had strong effect on the quality of grown layers. The films on copper contained quite a few defects including cracks and entrapped vacancies, whereas the films grown on nickel were pristine and defects free.
\end{abstract}

\section{Introduction}

The most prevalent form of carbon is graphite which consists of two dimensional sheets of $s p^{2}$ bonded atoms in a honeycomb lattice, graphene, which are held together by weak Van der Waals forces. Graphite possesses high degree of anisotropic properties owing to this different

S. Amini $(\varangle) \cdot$ H. Kalaantari · J. Garay · R. Abbaschian Department of Mechanical Engineering, University of California Riverside, Riverside, CA 92507, USA

e-mail:samini@engr.ucr.edu

A. A. Balandin

Department of Electrical Engineering, University of California Riverside, Riverside, CA 92507, USA in-plane and out-of-plane bonding characteristic. Due to weak forces in between, the graphite layers can be detached to form thin films. These sheets have the potential to be utilized as high temperature gaskets, electrodes in recharged batteries, adsorption substrates, and reinforcement phase in composite materials. It has been further established that single layer graphene (SLG) possess a large number of unique properties including the quantum Hall effect at room temperature [1], ambipolar field effect [2], high optical transparency [3], extremely high electron mobility [4], and capability for detection of single molecule via adsorption[5].

The abovementioned properties have stimulated active research of possible graphene device applications. From the practical point of view, some of the most interesting graphene properties are very high room temperature carrier mobility up to $27,000 \mathrm{~cm}^{2} \mathrm{~V}^{-1} \mathrm{~s}^{-1}$ [2], and high thermal conductivity around $5000 \mathrm{~W} \mathrm{mK}^{-1}$ at room temperature for large suspended graphene flakes [6]. The enhanced current and heat conduction properties are expected to be beneficial for electronic, interconnect and thermal management applications. It has also been demonstrated that graphene devices can operate at very low-levels of the electronic flicker noise, which is important for applications in sensors and communications [7].

Graphite nano films have been produced via chemical or mechanical methods. Chemical techniques are generally based on the intercalation of graphite [8]. In this method, an intercalation compound, such as $\mathrm{KC}_{8}$ which is synthesized by adding an alkali metal to high-purity graphite, is reacted with an aqueous solvent. Dissolution of potassium ions among graphite layers, and hydrogen gas evolution combined with ultrasonication lead to graphite exfoliation. The mechanical methods used for separation and isolation of graphite layers generally involve milling which employs 
repeated plastic deformation for detachment of graphene layers [9]. In 1999, Atomic Force Microscope was used to pattern small graphite pillars into highly oriented pyrolytic graphite (HOPG) by plasma etching [10]. This technique was later modified by transferring the pillars to a tipless cantilever which stamped down slabs of graphite films on a $\mathrm{SiO}_{2}$ substrate producing $10 \mathrm{~nm}$ ( $\sim 30$ layers) thin crystals [11]. In 2001, single nano-sized graphene sheets were prepared by electrophoretic deposition and heat-treatment of diamond nano-particles on HOPG substrate at $1600{ }^{\circ} \mathrm{C}$ [12]. At the time, the two dimensional crystal of SLG was thought to be thermodynamically unstable at finite temperatures. It was assumed that any atomic monolayer would have to roll or fold in order to achieve its lowest potential energy. Later by means of electron microscopy, small ripples within graphene layers were noticed. It is speculated that these imperfections on the surface of graphene are preventing it from rolling into nanotubes or other carbonic structures [13]. Eventually, isolation of SLG was accomplished by mechanical exfoliation utilizing a cellophane tape [2]. In this technique, the layers of graphite were repeatedly cleaved using a tape. Other techniques to synthesize SLG have also been utilized including epitaxial growth [14], Chemical Vapor Deposition (CVD) [15], chemically derived graphene from graphite oxide [16], and high pressure-high temperature (HPHT) growth [17].

The mechanical exfoliation, while suitable for laboratory research, is unlikely to become practical for commercial applications. The graphene growth by CVD is promising but still has a number of problems related to control of the thickness and quality. The abovementioned techniques also have other drawbacks resulting from large equipment cost and long processing times [18]. More recently growth of SLG from a metal-carbon melt was introduced by authors [19]. The technique is based on dissolution of carbon atoms in a molten metal, followed by the melt cooling to allow growth of dissolved atoms on top of the melt as graphene layers. In this article, the recent results on the morphology of graphite grown within and on the surface of the melt are presented.

\section{Experimental procedures}

The melt growth technique basically rely on dissolving carbon atoms inside a molten metal at a certain temperature, and then allowing the dissolved carbon to grow as graphite films on top of the melt at lower temperatures. In this process, as described previously [19], nickel and copper were melted in contact with a carbon source at a given temperature to saturate with carbon at a concentration that was determined from the binary phase diagram of metal-carbon. Upon cooling, as the solubility of carbon in the molten metal decreased, the excess carbon formed on top of the melt as well as other sites within the melt. The floating graphite layer could be either skimmed or allowed to freeze on the metal. The $\mathrm{Cu}-\mathrm{C}$ system was selected because of the limited solubility of carbon in molten copper and no carbide formation has also been reported for this binary. The Ni-C system, on the other hand, consists of a simple eutectic reaction at $1356.5^{\circ} \mathrm{C}$ with limited terminal solubility of carbon in nickel. The samples were prepared by arc melting process, melting in resistance furnace, induction melting, and electromagnetic levitation (EML). The first two techniques have been reported previously [19]. For the induction melting, the apparatus consisted of a wound copper coil coupled to a $20 \mathrm{KW}$ powered $8 \mathrm{MHz}$ high frequency generators. A glass tube was inserted inside the coil through which a stream of purified $\mathrm{Ar} / \mathrm{He}$ was passed. Specific amounts of metal and graphite were melted inside a boron nitride crucible and kept for $30 \mathrm{~min}$ for complete alloying. The samples were then cooled down by shutting off the power. In order to investigate the effect of cooling rate on growth morphology of graphite in $\mathrm{Ni}-\mathrm{C}$ system, an EML apparatus was also utilized. For this process, the arc melted specimens, weighing approximately $1.5 \mathrm{~g}$, were placed in the levitation melting apparatus similar to the one previously described [20]. The samples were levitation melted and superheated to $1750{ }^{\circ} \mathrm{C}$ for about $15 \mathrm{~min}$, then cooled either by $\mathrm{Ar} / \mathrm{He}$ gas mixture or by quenching in a coolant media placed beneath the levitation coil.

The surfaces or cross sections of the as-solidified samples were then investigated with optical microscope, scanning electron microscope (SEM), and Raman Spectroscopy. Additional investigations were done by dissolving the metal substrate away and transferring the graphite films to a silicon wafer by the method previously reported [21]. To achieve this, a layer of poly methyl methacrylate (PMMA) was spin coated on the substrate (1800 rpm for $30 \mathrm{~s})$. Afterward, the metal substrate was etched away by a nitric acid solution (1:2) allowing the PMMA/carbonic layer to float on top of the solution. After placing the layer on a glass substrate, it was washed with isopropanol and water. The PMMA/graphite layer was subsequently transferred to a $\mathrm{Si} / \mathrm{SiO}_{2}$ wafer, and the film was annealed at $60{ }^{\circ} \mathrm{C}$ for $1 \mathrm{~h}$ to adhere firmly to the target substrate. The PMMA was then dissolved gradually with acetone. Finally, the wafer with the carbonic layers was washed with isopropanol and dried with nitrogen gas.

Raman spectroscopy was carried out using a Horiba Jobin-Yvon micro-Raman spectrometer. All spectra were excited with visible $(632.8 \mathrm{~nm}$ ) laser light (power $3.6 \mathrm{~mW}$ ) and collected in the backscattering configuration. The spectra were recorded with a 1800 lines $/ \mathrm{mm}$ grating. A $100 \times$ objective was used to focus the excitation laser light 
on different spots of the samples. As described elsewhere $[22,23]$, the most prominent features in the Raman spectra of graphitic materials are the $\mathrm{G}$ band $\left(\sim 1582 \mathrm{~cm}^{-1}\right)$, D band $\left(\sim 1350 \mathrm{~cm}^{-1}\right), \mathrm{D}^{\prime}$ band $\left(\sim 1620 \mathrm{~cm}^{-1}\right)$, and the 2D band $\left(\sim 2700 \mathrm{~cm}^{-1}\right)$. The $\mathrm{G}$ band is prominent for $s p^{2}$ carbon networks. In contrast, $s p^{3}$ (diamond) and $s p$ carbon (linear carbon chains) show characteristic features at $1333 \mathrm{~cm}^{-1}$ and in the range $1850-2100 \mathrm{~cm}^{-1}$, respectively. The $\mathrm{D}$ and $\mathrm{D}^{\prime}$ bands were used to investigate the formation of defects. These defect induced Raman features are not observed in highly crystalline graphite. The 2D (or $\mathrm{G}^{\prime}$ ) band corresponds to the overtone of the D band observed in all kinds of graphitic materials and exhibit a strong Raman band which appears in the range $2500-2800 \mathrm{~cm}^{-1}$ [22]. The evolution of the 2D band Raman signatures with the addition of each extra layer of graphene was used to accurately count the number of layers. In addition, an estimate on the number of layers was obtained from analysis of $I_{\mathrm{G}} / I_{2 \mathrm{D}}$ ratio $[22,24]$.

\section{Results and discussion}

The growth mechanisms of graphite inside molten metals have been extensively investigated [25-27]. Most of these investigations have focused on the $\mathrm{Fe}-\mathrm{C}$ system (cast iron) due to diversity of graphite morphologies and their pronounced influence on the properties of industrially important alloys. Several graphite morphologies reported for $\mathrm{Fe}-\mathrm{C}$ include flakes, spheroids, compacted and star-like graphite [28]. Figure 1 shows different graphite morphologies in $\mathrm{Ni}-\mathrm{C}$ system solidified at various cooling rates and compositions. The microstructure of a hypo eutectic composition $(\mathrm{Ni}+1.35 \mathrm{wt} \% \mathrm{C})$ which was induction melted and cooled in Ar atmosphere at a rate of $22{ }^{\circ} \mathrm{C} \mathrm{s}^{-1}$ is shown in Fig. 1a. The microstructure consists of nickel dendrites (bright phase) with a fully divorced eutectic (darker phase) in the boundaries. The divorced eutectic is the characteristic of hypoeutectic alloys with relatively low solute composition [29]. Figure 1b shows the microstructure close to the surface of a hyper eutectic alloy $(\mathrm{Ni}+3.05 \mathrm{wt} \% \mathrm{C})$ which was electromagnetically levitated and cooled with $\mathrm{He}$ gas with cooling rate of $34{ }^{\circ} \mathrm{C} \mathrm{s}^{-1}$. The microstructure consists of primary Kish flakes and fine coupled eutectic of nickel and graphite. The primary flakes are approximately $200 \mu \mathrm{m}$ long and $10 \mu \mathrm{m}$ thick. The Kish graphite flakes are different from the eutectic flakes in their coarse forms and smooth surfaces. The denuded nickel envelopes (nickel-rich regions) covered the Kish graphite as a bright phase. The nickel envelopes could be either formed during solidification around the primary graphite or after solidification by diffusion of carbon atoms in the solid [30]. Figure 1c shows a
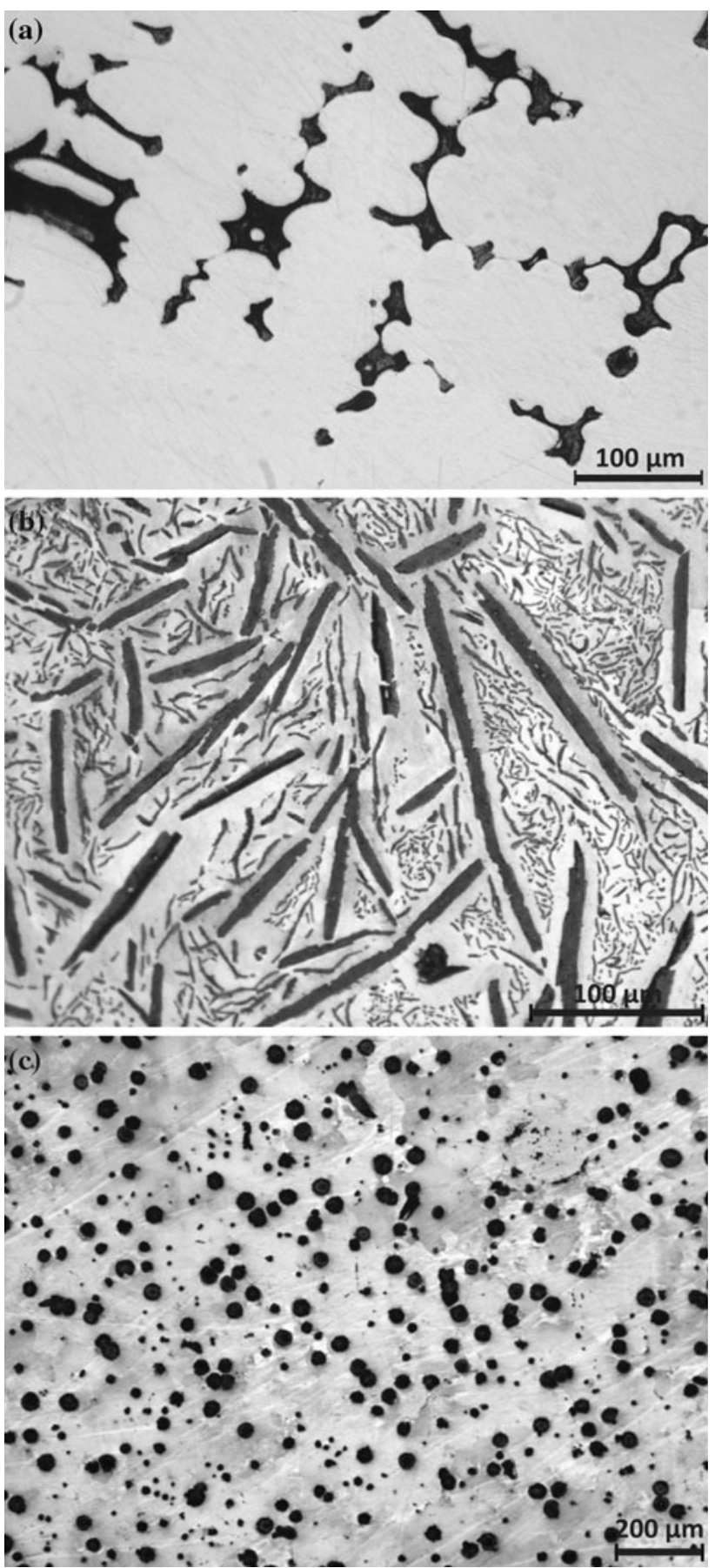

Fig. 1 Effect of cooling rate and composition on the microstructure of $\mathrm{Ni}-\mathrm{C}$ alloys. a Hypo eutectic alloy $(\mathrm{Ni}+1.35 \mathrm{wt} \% \mathrm{C})$ induction melted and cooled in Ar atmosphere (cooling rate $22{ }^{\circ} \mathrm{C} / \mathrm{s}$ ). b Hyper eutectic alloy $(\mathrm{Ni}+3.05 \mathrm{wt} \% \mathrm{C})$, electromagnetically levitated and cooled with $\mathrm{He}$ gas (cooling rate $34{ }^{\circ} \mathrm{C} / \mathrm{s}$ ). c Hyper eutectic alloy $(\mathrm{Ni}+3.05 \mathrm{wt} \% \mathrm{C})$, electromagnetically levitated and quenched in oil

hyper eutectic alloy $(\mathrm{Ni}+3.05 \mathrm{wt} \% \mathrm{C})$ quenched in oil. No couple growth could be observed and the microstructure solely consisted of spherical graphite. It has already been reported that with increasing cooling rate a transition 
from lamellar to spheroids will occur [31]. However, the exact mechanisms of nucleation and growth are not fully understood.

In contrast with nickel system, no graphite formation within copper was observed since the solubility of carbon in molten copper is very limited. The graphite formation for copper was mostly limited to the free surfaces or metalcrucible boundaries.

While numerous studies have been carried out on the growth of graphite flakes inside the molten metal, yet less attention has been given on growth of graphite films on the molten metal. Direct visual observation of graphite growth on top of the melt was utilized by means of a portable microscope. Figure 2 shows video frames of a hypereutectic Ni-C melt which is cooled from uniform liquid to liquid + graphite region in phase diagram and once more heated up. Frames 1-4 demonstrate the cooling while the frames 5-6 show the heating process.
The frames show two distinct regions of different brightness, with the brighter area showing graphite layer. The graphite is completely dissolved in higher temperature (frame 1). Nevertheless, there are observations that propose the phase in frame 1 is not purely bare metal and it is possible that thin graphitic shell is present over the entire surface [32]. As the melt is cooled down to lower temperature (liquid + graphite region) the excess amount of carbon grows as graphite patches on the surface (frame 2-3). With continuous cooling, those patches grow until they cover the entire surface (frame 4). This process is reversible as upon heating, the graphitic layer will dissolve back into the melt (frame 5-6). Figure 3 shows a graphite film which has been skimmed from the surface of molten nickel by means of a boron nitride substrate during the growing stage (frame 3 in Fig. 2). The boundaries show polygonal shape which is the characteristic of faceted growth of graphite.
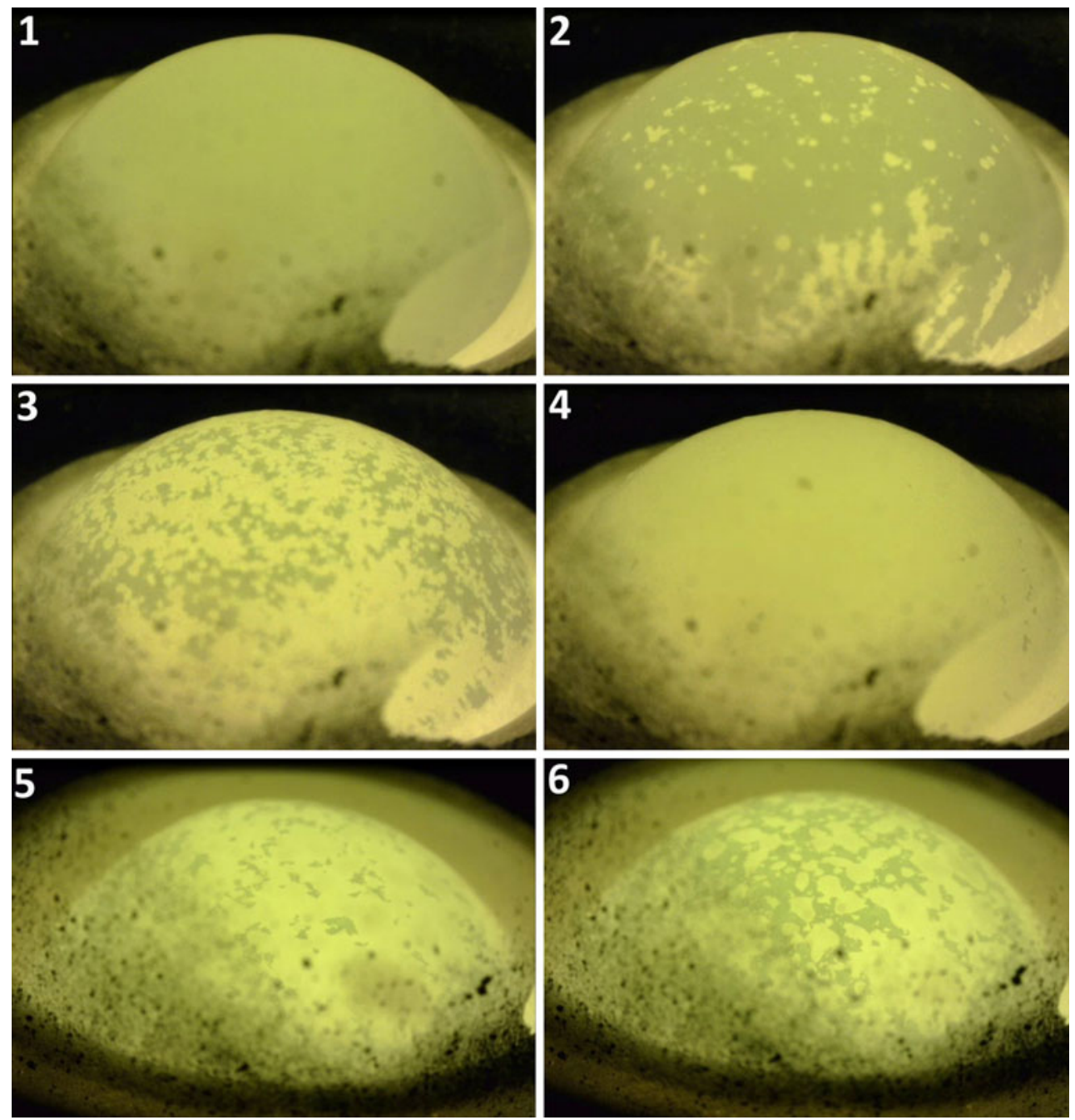

Fig. 2 Video frames of graphite growth on top of the melt: Frames 1-4 demonstrate the melt cooling and subsequent growth of graphite films, frames 5-6 show the melt heating and succeeding graphite dissolution (the brighter phase is graphite) 


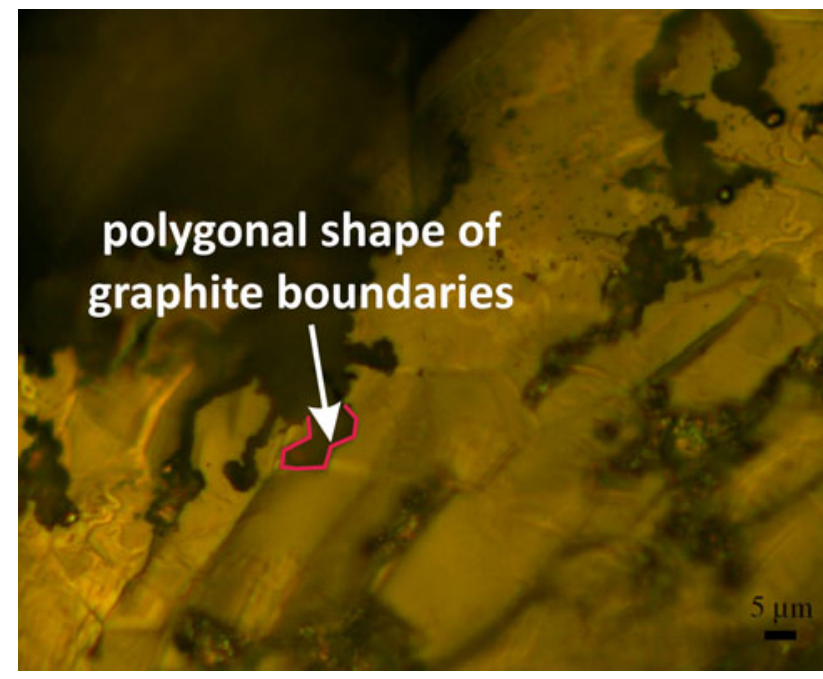

Fig. 3 Skimmed graphite film from the surface of molten nickel during early growth (Frame 3 in Fig. 2)

Figure 4a shows the graphite layer which has been formed on top of nickel upon complete solidification of the $\mathrm{Ni}+2.35 \mathrm{wt} \% \mathrm{C}$ melt. The film has a specific morphology of smooth surface areas separated from each other by outof-plane faceted wrinkles. The structure at the joint between the wrinkles is more complicated, but the faceted structure is still evident. Figure $4 \mathrm{~b}$ shows typical Raman Spectra of graphite in smooth areas and wrinkles. The two spectra, which have been shifted slightly for the ease of visualization, are basically identical. The spectra show an intense $\mathrm{G}$ band as well as an asymmetric $2 \mathrm{D}$ band. No $\mathrm{D}$ $\left(\sim 1350 \mathrm{~cm}^{-1}\right)$ and $\mathrm{D}^{\prime}\left(\sim 1620 \mathrm{~cm}^{-1}\right)$ bands could be noticed in either spectrum. As discussed earlier, D and $\mathrm{D}^{\prime}$ bands are indicative of defects. Similar Raman Spectroscopy was carried out in different spots of the graphite and the results were identical. The Raman features shown in Fig. $4 \mathrm{~b}$ were found to be similar to those of bulk crystalline graphite reported in literature [22]. By comparing the smooth areas and wrinkles Raman features, it is evident that the structure of wrinkles is identical to flat areas. In fact, the wrinkles are part of the graphite film and hold the same crystal structure of flat areas.

The wrinkled feature of the graphite layers is believed to be due to the accommodation of the thermal expansion coefficient difference between the metal substrate and the graphite layer [33]. Different thermal expansion coefficient of nickel and graphite will give rise to a larger lateral contraction of metal substrate than that of graphite film. As a result, a compressive biaxial stress will develop on the graphite layer which consequently leads to the formation of triangular folds in the film, schematically shown in Fig. 5. It is apparent that as the graphite films deform, they shift upward and they lose their week out-of-plane bonds in some areas and detached from each other. An example is (a)
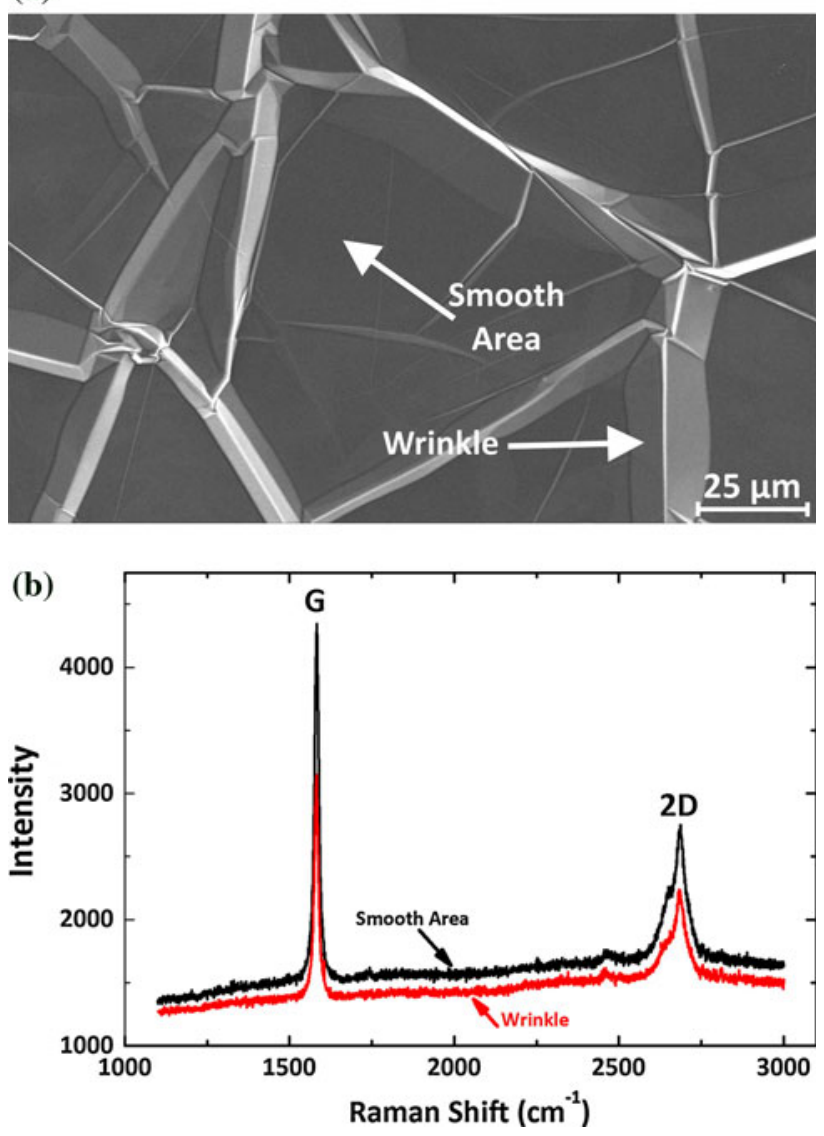

Fig. 4 a SEM Photo (Secondary Electron image) of thick graphite layer formed on top of nickel and $\mathbf{b}$ Raman spectra of smooth areas and wrinkles

shown in Fig. 6 as a wrinkle was cross sectioned using Focused Ion Beam. The hollow spaces which have been shown schematically in Fig. 5 can be observed clearly in the cross section of Fig. 6 .

During the cooling, each individual wrinkles in the graphite layer contributes to the strain accommodation. The difference in thermal strain of graphite layer and nickel is $\Delta \varepsilon=\Delta T\left(\alpha_{\mathrm{Ni}}-\alpha_{\mathrm{gr}}\right)$, where $\alpha_{\mathrm{Ni}}$ and $\alpha_{\mathrm{gr}}$ are the bulk and in-plane thermal expansion coefficients of nickel and graphite and can be found as functions of temperature in literature [34-36]. For calculating the thermal strain, the mean values of thermal expansion coefficients were selected for nickel and graphite which are $18.04 \times 10^{-6}$ and $0.5 \times 10^{-6} \mathrm{~K}^{-1}$, respectively. The total strain needed to be accommodated is then $\Delta \varepsilon=\left(T_{\text {eutectic }}-T_{\text {ambient }}\right)\left(\alpha_{\mathrm{Ni}}-\alpha_{\mathrm{gr}}\right)=0.023$. Based on the examining of different wrinkles on the surface, the lateral size and roof angle can be considered approximately $L=10 \mu \mathrm{m}$ and $\varphi=120^{\circ}$, respectively. Based on the wrinkles geometry, the amount of layered material shifted from adjacent regions toward the wrinkle approximately corresponds to $\Delta L=2(L / 2 \cos 30)-L=1.55 \mu \mathrm{m}$. 

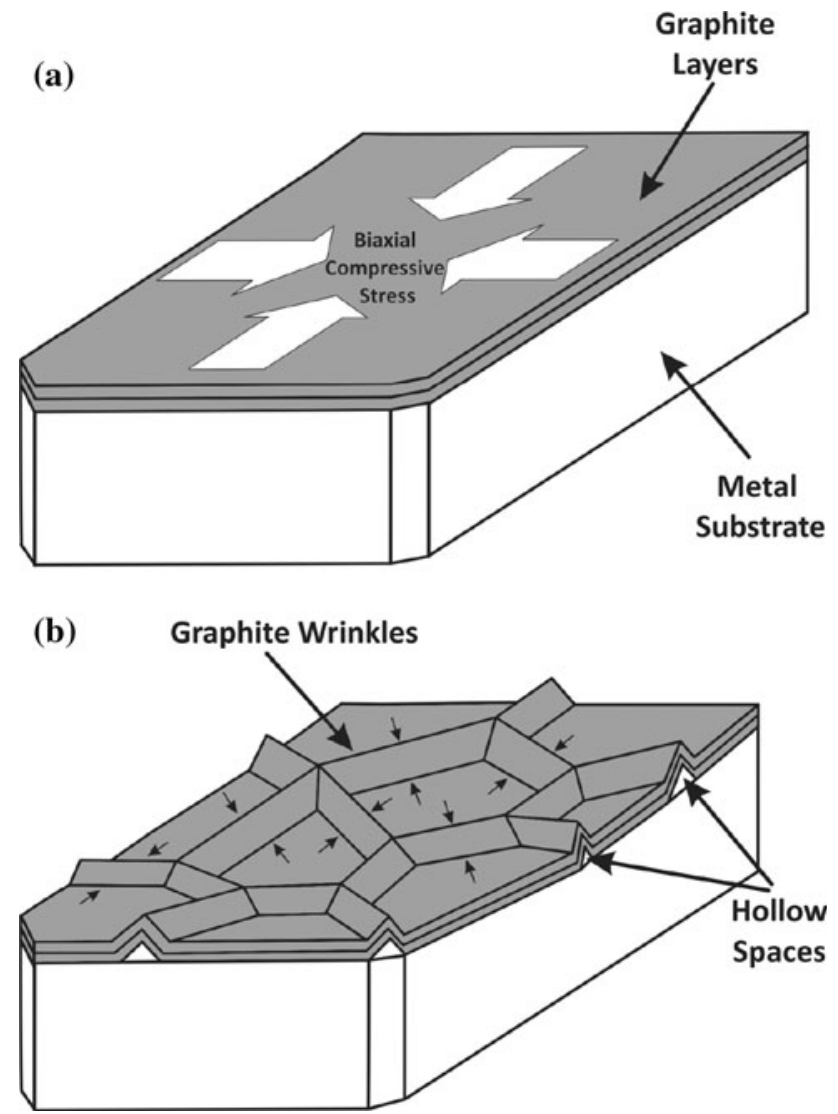

Fig. 5 Schematic of wrinkle formation on graphite layers due to thermal expansion coefficient mismatch

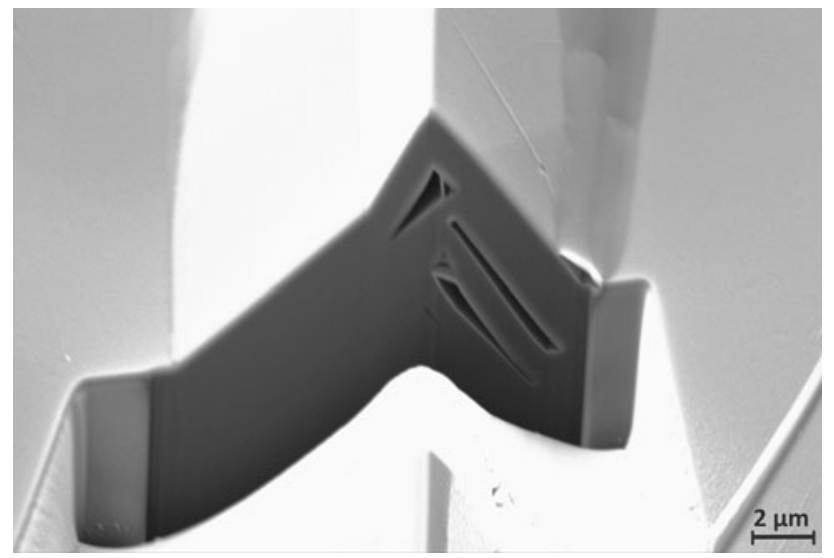

Fig. 6 Cross section of a wrinkle by Focused Ion Beam cutting

To completely accommodate a $2.3 \%$ strain, an array of wrinkles should be formed with spacing of $r=\Delta L /$ $\Delta \varepsilon=67 \mu \mathrm{m}$. This value is as the same order of magnitude $(50 \mu \mathrm{m})$ which is observed in Fig. 4.

Figure 7 shows the SEM photo of electron-transparent graphitic layers on metallic substrate, indicating that the layers are thin enough to serve as window for 5 and $10 \mathrm{keV}$ electrons to pass along. Additional details of one of the
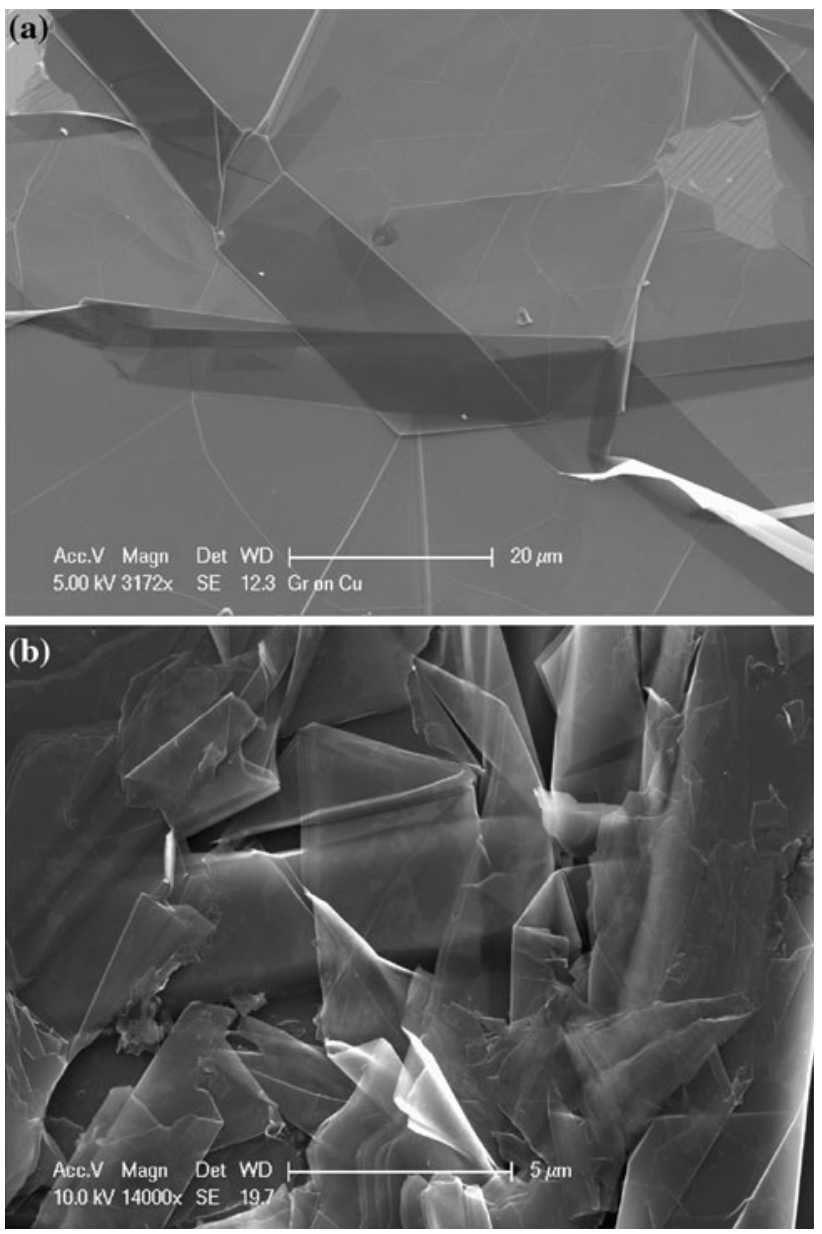

Fig. 7 SEM Photo (Secondary Electron image) of electronically transparent thin graphite films on a copper and $\mathbf{b}$ nickel

grown islands, which were transferred to silicon wafer, are shown in Fig. 8a. Different color contrasts in this film indicate areas which are rather thick, but connected by thinner regions, similar to that shown schematically in Fig. 8b. As a graphitic layer nucleates on the melt, it expands laterally and normally by $2 \mathrm{D}$ nucleation and faceted growth, in a manner similar to that observed for $2 \mathrm{D}$ nucleation and growth of faceted materials [37]. The Raman spectrum shown in Fig. 8c represents the region which labeled as "few layer graphene" on the film. The $I_{\mathrm{G}} /$ $I_{2 \mathrm{D}}$ ratio is the evidence that the existing flake has few graphene layers. The spectrum features a symmetric 2D band and intense $\mathrm{D}$ and $\mathrm{D}^{\prime}$ bands. The symmetric $2 \mathrm{D}$ band denotes the existence of turbostratic graphite (without $\mathrm{ABAB}$ stacking). The intense $\mathrm{D}$ and $\mathrm{D}^{\prime}$ peaks show layers with high amount of defects. FLG were also observed at the edges of graphite films [19].

It is conceivable that defect formation in the formed layers on copper is due to high cooling rate of copper. The high thermal diffusivity of copper (nearly five times of nickel) will lead to high cooling rate and consequently 
(a)

(b)

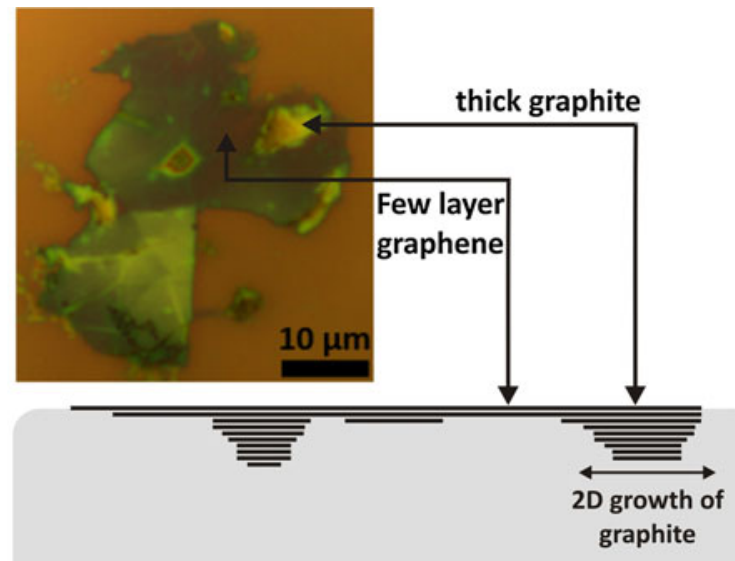

\section{Metal}

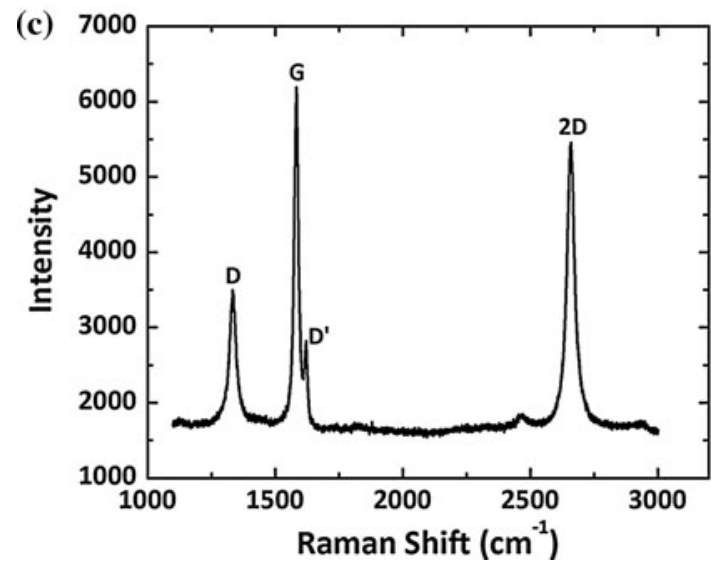

Fig. 8 a Optical Microcopy of a graphite film formed on copper and then transferred to $\mathrm{Si} / \mathrm{SiO} 2$, b schematic of 2D growth of graphite, c Raman spectrum of the spot labeled as few layer graphene in (a)

more defect formation including cracks and entrapped vacancies [38]. For comparison, Raman spectrum of FLG on nickel is being shown in Fig. 9a. The absence of D and $\mathrm{D}^{\prime}$ bands represents defect free FLG on nickel. The lower thermal diffusivity of nickel will result in lower cooling rate and subsequent pristine graphene films formation. The deconvoluted 2D band of spectrum, shown in Fig. 9b, reveals two Lorentzian peaks $\left(\Delta \omega=28 \mathrm{~cm}^{-1}\right)$, indicating four-layer graphene.

Interestingly, nickel substrate was found to have inactive Raman spectrograph, lending itself as an appropriate substrate for direct characterization of graphene layers without the need for transferring the layers to silicon wafer. Pristine SLG layers were observed in various locations on nickel substrate. The Raman spectrum of one such layer is shown in Fig. 10. The spectrum shows $G$ band at $1582 \mathrm{~cm}^{-1}$ and a $2 \mathrm{D}$ band at $2660 \mathrm{~cm}^{-1}$. The $I_{\mathrm{G}} / I_{2 \mathrm{D}}$ ratio is 3.7 and $2 \mathrm{D}$ band is symmetrical, which is similar to
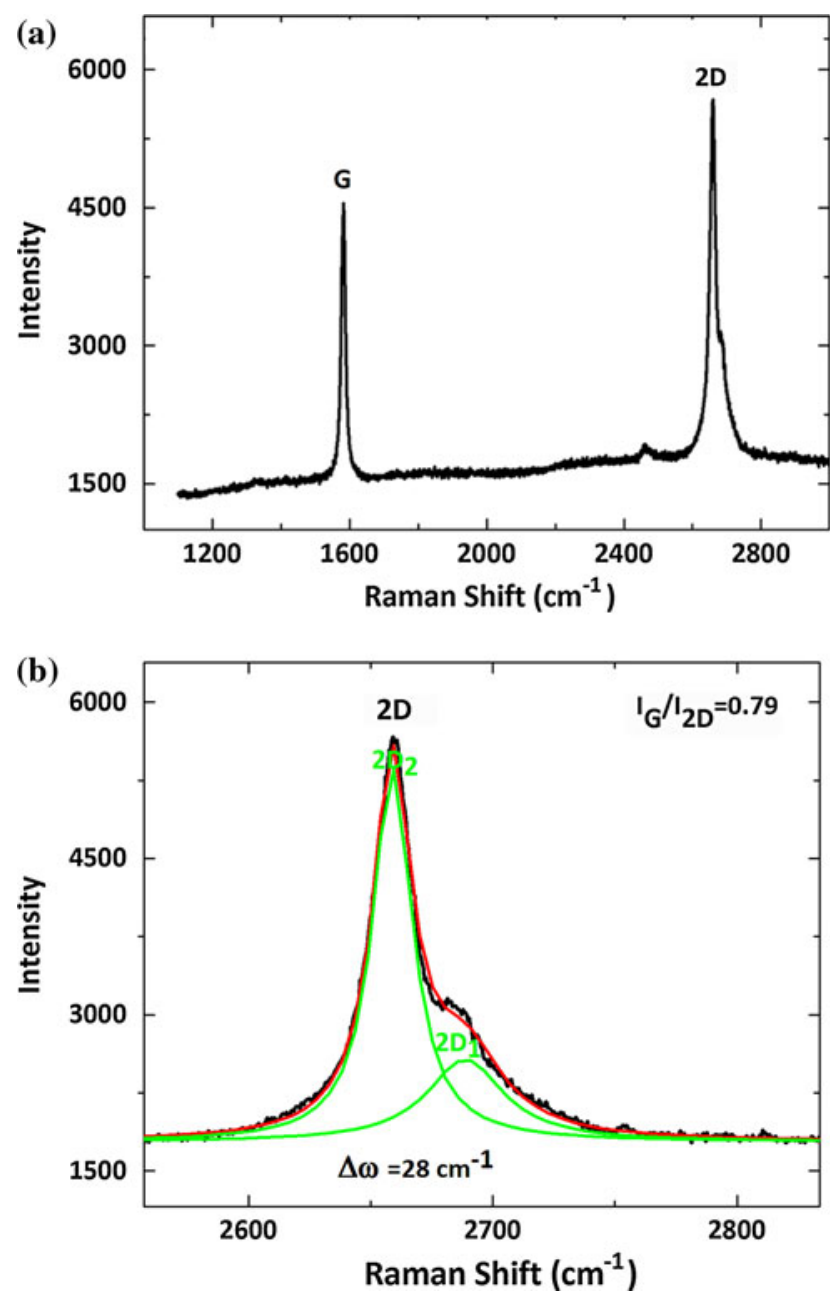

Fig. 9 a Raman spectrum of FLG formed on nickel and b deconvolution of $2 \mathrm{D}$ band

reported features for SLG. The absence of the D and $\mathrm{D}^{\prime}$ peaks is indicative that the formed SLG is defect free.

The amount of graphite forming on the melt and its characteristic will strongly depend on the amount of carbon dissolved in the melt and the solubility limit of carbon in the liquid as well as cooling conditions employed. For this investigation, the two alloy systems of $\mathrm{Cu}-\mathrm{C}$ and $\mathrm{Ni}-\mathrm{C}$ show extensive differences in solubility limit. It appears that the Ni-C system is more conducive to the formation of large and defect free layers.

\section{Conclusion}

In this research, a new technique for growing graphite nano crystals was utilized. The technique involved dissolving carbon in a molten metal at a specified temperature and then allowing the dissolved carbon to nucleate and grow on 
(a)
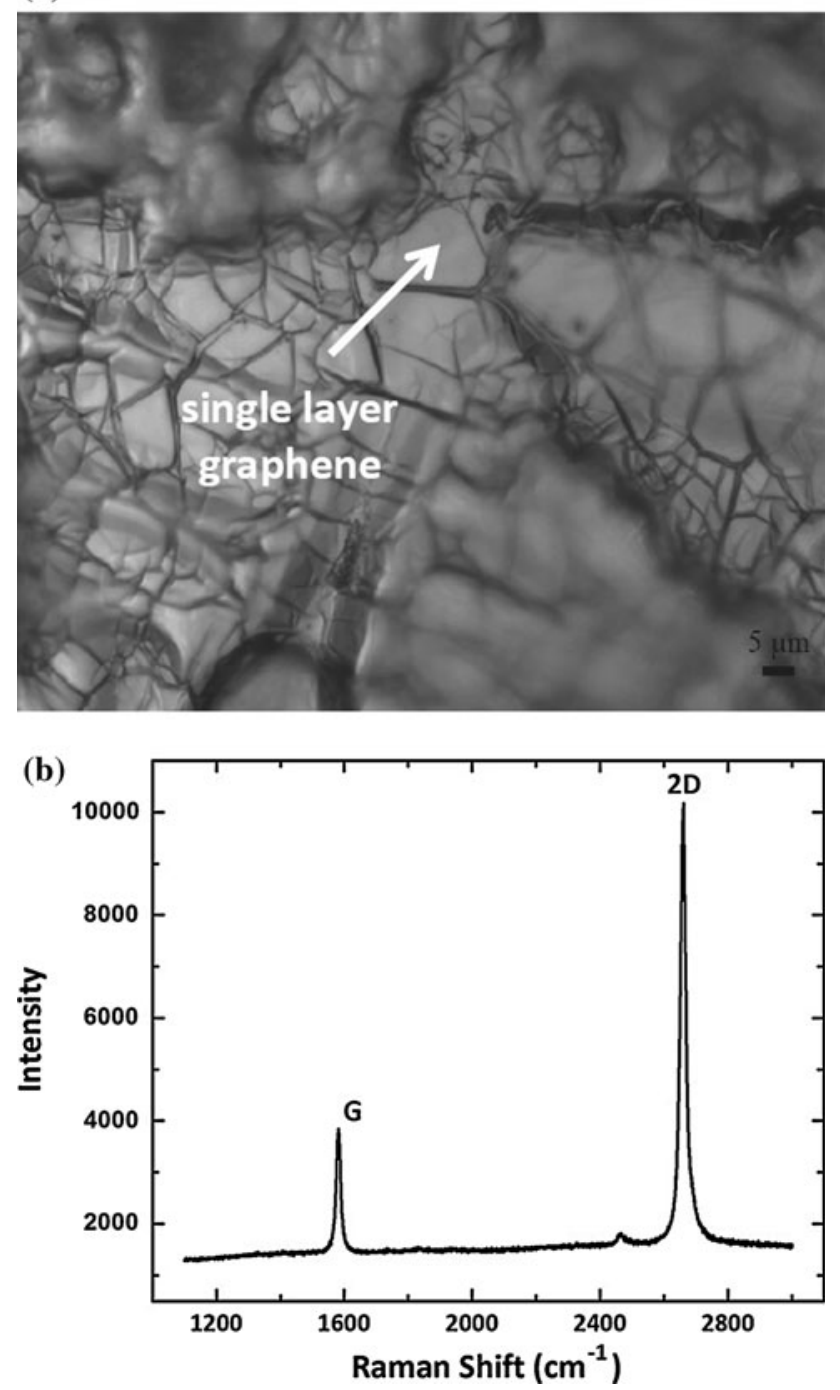

Fig. 10 a Optical Microcopy of SLG formed on nickel, b its Raman Spectrum

top of the melt at a lower temperature. Detailed optical microscopy, Raman spectroscopy, and scanning electron microscopy were utilized to characterize the consequential grown layers. It was found that the different morphologies including thick graphite, FLG, and SLG could form with the technique. The grown films consisted of faceted wrinkles which the layers conserved their continuity over them. The formation of these wrinkles is believed to be due to thermal expansion coefficient mismatch between metal substrate and graphite. Although the wrinkles were not dependent on the type of metal substrate, the substrate had strong effect on the quality of grown layers. The layers formed on copper showed extensive defects while the ones on nickel were highly ordered crystal structure free of defects. Pristine FLG and SLG were successfully grown on nickel substrate.

Open Access This article is distributed under the terms of the Creative Commons Attribution Noncommercial License which permits any noncommercial use, distribution, and reproduction in any medium, provided the original author(s) and source are credited.

\section{References}

1. Novoselov KS, McCann E, Morozov SV, Falko VI, Katsnelson MI, Zeitler U, Jiang D, Schedin F, Geim AK (2006) Nat Phys 2:177

2. Novoselov KS, Geim AK, Morozov SV, Jiang D, Zhang Y, Dubonos SV, Grigorieva IV, Firsov AA (2004) Science 306:666

3. Nair RR, Blake P, Grigorenko AN, Novoselov KS, Booth TJ, Stauber T, Peres NMR, Geim AK (2008) Science: 1156965

4. Han M, Ozyilmaz B, Zhang Y, Jarillo-Herero P, Kim P (2007) Phys Stat Solid (b) 244:4134

5. Schedin F, Geim AK, Morozov SV, Hill EW, Blake P, Katsnelson MI, Novoselov KS (2007) Nat Mater 6:652

6. Balandin AA, Ghosh S, Bao W, Calizo I, Teweldebrhan D, Miao F, Lau CN (2008) Nano Lett 8:902

7. Lin Y-M, Avouris P (2008) Nano Lett 8:2119

8. Viculis LM, Mack JJ, Kaner RB (2003) Science 299:1361

9. Chen Y, Conway MJ, Fitz Gerald JD, Williams JS, Chadderton LT (2004) Carbon 42:1543

10. Lu X, Yu M, Huang H, Ruoff RS (1999) Nanotechnology 10:269

11. Zhang Y, Small JP, Pontius WV, Kim P (2005) Appl Phys Lett 86:073104

12. Affoune AM, Prasad BLV, Sato H, Enoki T, Kaburagi Y, Hishiyama Y (2001) Chem Phys Lett 348:17

13. Geim AK, Novoselov KS (2007) Nat Mater 6:183

14. Berger C, Song Z, Li T, Li X, Ogbazghi AY, Feng R, Dai Z, Marchenkov AN, Conrad EH, First PN, de Heer WA (2004) J Phys Chem B 108:19912

15. Yu Q, Lian J, Siriponglert S, Li H, Chen YP, Pei S-S (2008) Appl Phys Lett 93:113103

16. Stankovich S, Dikin DA, Piner RD, Kohlhaas KA, Kleinhammes A, Jia Y, Wu Y, Nguyen ST, Ruoff RS (2007) Carbon 45:1558

17. Parvizi RF, Teweldebrhan D, Ghosh S, Calizo I, Balandin AA, Zhu H, Abbaschian R (2008) Micro Nano Lett IET 3:29

18. Taghioskoui M (2009) Mater Today 12:34

19. Amini S, Garay J, Liu G, Balandin AA, Abbaschian R (2010) J Appl Phys 108:094321

20. Kim D, Abbaschian R (2000) J Phase Equilib 21:25

21. Reina A, Jia X, Ho J, Nezich D, Son H, Bulovic V, Dresselhaus MS, Kong J (2008) Nano Lett 9:30

22. Ferrari AC, Meyer JC, Scardaci V, Casiraghi C, Lazzeri M, Mauri F, Piscanec S, Jiang D, Novoselov KS, Roth S, Geim AK (2006) Phys Rev Lett 97:187401

23. Gupta A, Chen G, Joshi P, Tadigadapa S, Eklund (2006) Nano Lett 6:2667

24. Calizo I, Bejenari I, Rahman M, Liu G, Balandin AA (2009) J Appl Phys 106:043509

25. Double DD, Hellawell A (1974) In: Lux B, Minkoff I, Mollard F (eds) Metallurgy of cast iron. Gorgi, Switzerland

26. Minkoff I, Lux B (1974) In: Lux B, Minkoff I, Mollard F (eds) Metallurgy of cast iron. Gorgi, Switzerland

27. Lux B, Vendl A, Hahn H (1981) Cryst Res Technol 16:531

28. Elliott R (1988) Cast iron technology. Butterworth and Co, London 
29. Dantzig JA, Rappaz M (2009) Solidification EPFL Press. Lausanne, Switzerland

30. Lux B, Mollard F, Minkoff I (1974) In: Lux B, Minkoff I, Mollard F (eds) Metallurgy of cast iron. Gorgi, Switzerland

31. Brigham RJ, Purdy GR, Kirkaldy JS (1967) J Cryst Growth 20-24:161

32. Winder SM, Liu D, Bender JW (2006) Carbon 44:3037

33. Spiecker E, Schmid AK, Minor AM, Dahmen U, Hollensteiner S, Jäger W (2006) Phys Rev Lett 96:086401
34. Kollie TG (1977) Phys Rev B 16:4872

35. Mounet N, Marzari N (2005) Phys Rev B 71:205214

36. Pierson HO (1993) Handbook of carbon, graphite, diamond and fullerenes: properties, processing, and applications. Noyes Publications, Park Ridge

37. Peteves S, Abbaschian R (1991) Metall Mater Trans A 22:1271

38. Thrower PA (1969) Chemistry and physics of carbon. Marcel Dekker, New York 